\title{
Molecular Detection of Mutations in the Propeller Domain of Kelch 13 and pfmdr1 Copy Number Variation in Plasmodium falciparum Isolates from Thailand Collected from 2002 to 2007
}

\author{
Chaiyaporn Chaisatit, ${ }^{1}$ Piyaporn Sai-ngam, ${ }^{1}$ Sasikanya Thaloengsok, ${ }^{1}$ Sabaithip Sriwichai, ${ }^{1}$ Krisada Jongsakul, ${ }^{1}$ Mark Fukuda, ${ }^{2}$ \\ Michele Spring, ${ }^{1}$ Mariusz Wojnarski, ${ }^{1}$ Norman Waters, ${ }^{1}$ Paphavee Lertsethtakarn, ${ }^{1}$ and Brian A. Vesely ${ }^{1 *}$ \\ ${ }^{1}$ Department of Bacterial and Parasitic Diseases, Armed Forces Research Institute of Medical Sciences, Bangkok, Thailand; ${ }^{2}$ Department of \\ Retrovirology, Armed Forces Research Institute of Medical Sciences, Bangkok, Thailand
}

\begin{abstract}
We determined the prevalence of Kelch 13 mutations and pfmdr1 copy number in samples collected from the Thailand-Myanmar border, the Thailand-Cambodia border, and southern Thailand from 2002 to 2007. C580Y was the most prevalent in Trat (Thailand-Cambodia border) and Ranong (Thailand-Myanmar border) at 42\% (24/57) and 13\% (6/48), respectively. Less predominant mutations were also identified including R539T (7\%, 4/57) and Y493H (2\%, 1/57) in Trat, P574L (6\%, 3/48) and P553L (2\%, 1/48) in Ranong, and N537I and D452E (7\%, 1/15) in Sangkhlaburi (Thailand-Myanmar border). Samples from Mae sot $(33 \%, 11 / 33)$ harbored the highest percentage of multiple pfmdr1 copies, followed by Trat (18\%, 10/57), Chiang Dao in 2003 (13\%, 4/30), Phang Nga (5\%, 2/44), and Chiang Dao in 2002 (4\%, 1/26). This retrospective study provides geographic diversity of $\mathrm{K} 13$ and pfmdr1 copies and the emergence of these molecular markers in Thailand, an important background information for future surveillance in the region.
\end{abstract}

Artemisinin-based combination therapy (ACT) has been used as the first line of treatment of an uncomplicated Plasmodium falciparum infection in Thailand since $1995 .^{1}$ Extensive use of ACTs in the Greater Mekong Subregion (GMS) has raised concerns of independent emergence or spread of ACT-resistant $P$. falciparum. Clinical treatment failure attributed to artemisinin (ART) resistance in $P$. falciparum was first confirmed in Cambodia along the Thailand-Cambodia border in 2007 . $^{2,3}$

Validated molecular markers have been widely used as identification and prediction tools for the emergence of drug-resistant $P$. falciparum in new locations. Clinical ART resistance is defined as a delayed parasite clearance, a parasite clearance half-life $\left(\mathrm{PC}_{1 / 2}\right)$, which is greater than 5 hours. ${ }^{4}$ Mutations in the Kelch 13 (K13) propeller domain were shown to be strongly associated with delayed parasite clearance making it a good molecular marker for predicting clinical ART resistance. Nonsynonymous K13 mutations segregate into two distinct geographical areas where C580Y, R539T, $\mathrm{Y} 493 \mathrm{H}$, and I543T are commonly found in Cambodia, Vietnam, and Laos, respectively, whereas F446I, N458Y, P574L, and $\mathrm{R} 561 \mathrm{H}$ are more specific to the western part of Thailand, Myanmar, and China, respectively. ${ }^{5}$ An increase in pfmdr1 copy number has been linked to $P$. falciparum diminished susceptibility to mefloquine. ${ }^{6}$ Therefore, the surveillance of these molecular markers is useful to track the rise and spread of antimalarial drug resistance in $P$. falciparum.

This study was approved by the Thai Ministry of Public Health (MoPH, Ref. no. 60/2559). Participants from studies in which the samples were collected from provided written inform consents for future use of their blood. This study aimed to determine the presence and track the emergence of $\mathrm{K} 13$ and Pfmdr1 copy number over two decades. A total of 253 $P$. falciparum infected blood samples collected from six provinces in Thailand from 2002 to 2007 were assessed for a prevalence of $\mathrm{K} 13$ mutations and pfmdr1 copy number. Of these

\footnotetext{
*Address correspondence to Brian A. Vesely, Department of Bacterial and Parasitic Diseases, Armed Forces Research Institute of Medical Sciences, 315/6 Rajvithi Rd., Bangkok 10400,
} Thailand. E-mail: brian.vesely.mil@afrims.org samples, 152 were collected along the Thailand-Myanmar border (56 samples from Chiang Dao in 2002 and 2003, 33 samples from Mae Sot in 2004, 48 samples from Ranong in 2004, and 15 samples from Sangkhlaburi in 2007), whereas in 2005, 57 and 44 samples were collected from Trat, along the Thailand-Cambodia border, and Phang nga in southern Thailand, respectively. Genomic DNA was extracted from ethylenediaminetetraacetic acid (EDTA) whole blood using EZ1 DNA blood kit with automated EZ1 Advanced XL purification system (QIAGEN, Valencia, CA). Plasmodium speciation was confirmed by using multiplex real-time polymerase chain reaction (PCR) assay. ${ }^{7}$ Mutations in the propeller domain of $P$. falciparum $\mathrm{K} 13$ were determined by nested PCR and DNA sequencing. ${ }^{4}$ Pfmdr1 copy number was determined by TaqMan real-time PCR methods by using $\beta$-tubulin as a housekeeping gene and calculated using $2^{-\Delta \Delta C t}$ method. ${ }^{6}$

K13 mutations were successfully determined in 251/253 samples (99.2\%). Geographic distribution of K13 propeller domain polymorphisms is shown in Figure 1. No K13 mutations were found in samples from Chiang Dao and Mae Sot. The highest proportion of ART-resistance confirmed K13 mutations were found in Trat, with C580Y as the most common mutation (42\%, 24/57), followed by R539T (7\%, 4/57) and $\mathrm{Y} 493 \mathrm{H}(2 \%, 1 / 57)$. C580Y was also detected in Ranong and Phang Nga, which are geographically distant from the Thailand-Cambodia border, at 13\% (6/48) and 2\% (1/44), respectively. The K13 mutations $\mathrm{R} 539 \mathrm{~T}$ and $\mathrm{Y} 493 \mathrm{H}$ were only found in Trat, whereas P574L and P553L, ARTresistance associated K13 mutations were found in Ranong at $6 \%(3 / 48)$ and $2 \%(1 / 48)$, respectively. Although the sample size for Sangkhlaburi was small, N537I and D452E were observed (each at $7 \%, 1 / 15)$.

The pfmdr1 copy number was successfully determined in $208 / 253$ samples (82.2\%). The highest proportion of samples harboring multiple pfmdr 1 copies was found in samples from Mae Sot $(33 \%, 11 / 33)$, followed by Trat (18\%, 10/57), Chiang Dao in 2003 (13\%, 4/30), Phang Nga (5\%, 2/44), and Chiang Dao in 2002 (4\%, 1/26). All samples from Ranong and Sangkhlaburi harbored a single pfmdr1 copy (Table 1).

The information of $\mathrm{K} 13$ propeller domain polymorphisms together with pfmdr1 copy number for 208 samples (82.2\%) are shown in Figure 2. An increase in samples harboring 


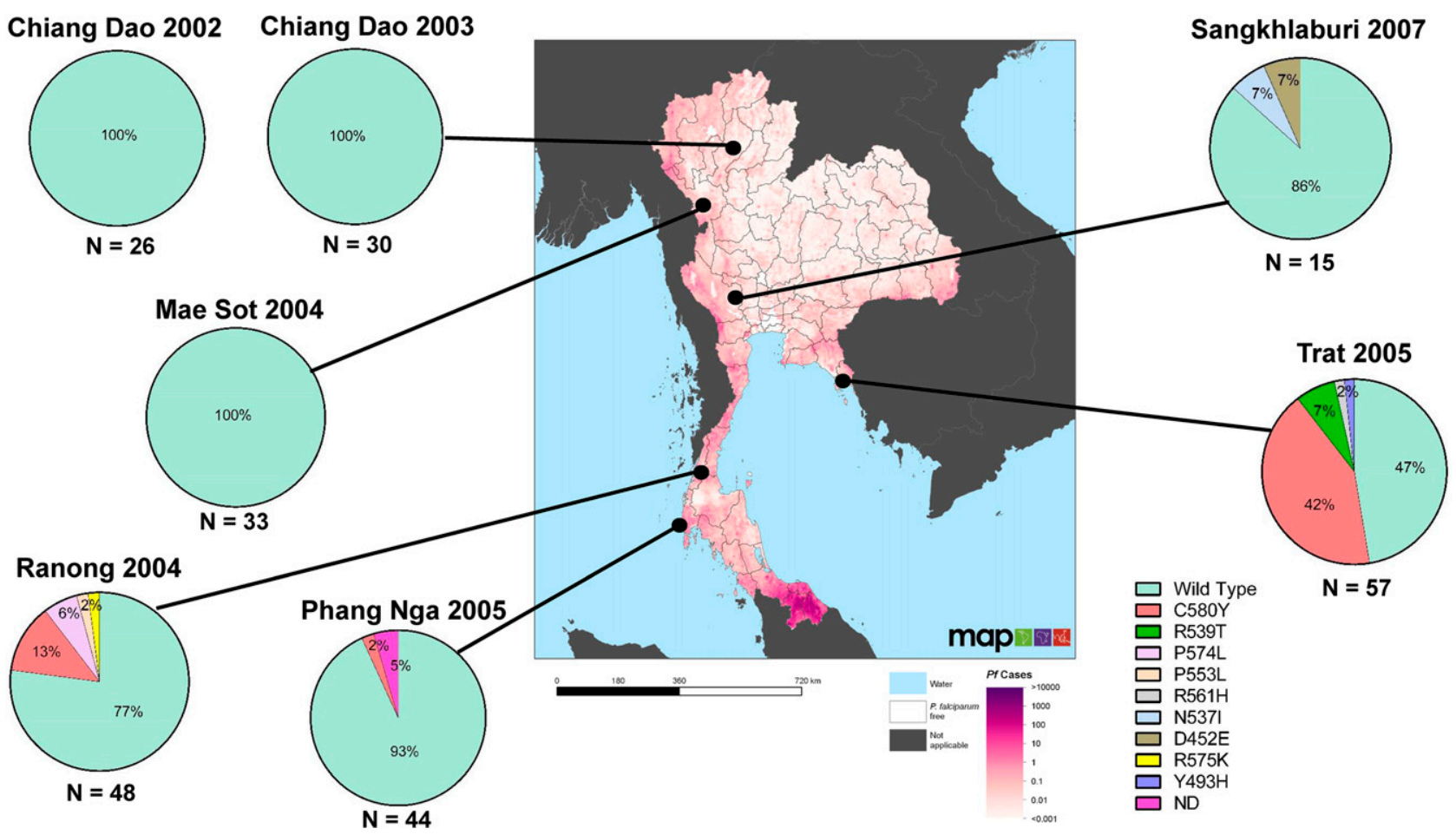

FIGURE 1. Geographic distribution of Plasmodium falciparum K13 mutations collected in six provinces in Thailand from 2002 to 2007 . The results are depicted on the spatial distribution of $P$. falciparum cases in Thailand in 2007 (Malaria Atlas Project). This figure appears in color at www.ajtmh.org.

multiple pfmdr1 copies with wild-type K13 was observed from 2002 to 2004 in Chiang Dao 2002, Chiang Dao 2003, and Mae Sot 2004. Nonsynonymous K13 mutations were first detected in 2004 and in 2005 and 2007, all with a single pfmdr1 copy except in Trat in 2005 with K13 mutations combined with either single or multiple copy of pfmdr1. Interestingly, multiple pfmdr1 copies with mutated K13 was observed only in samples from Trat.

Other studies have reported mutations and amplifications of candidate markers conferring antimalarial drug resistance along borders of Thailand with Myanmar, and Cambodia, and in southern Thailand. ${ }^{8,9}$ The earliest report of mutations in K13 in Thailand was in Trat in 1991, but those mutations were not associated with ART resistance as classified by the WHO. ${ }^{10}$ This study provides retrospective information on prevalence and distribution of $\mathrm{K} 13$ mutations in Thailand as early as 2002, and to date, the C580Y mutation found in Ranong is the earliest observation of C580Y mutation in Thailand. This finding is 3 years before other retrospective

TABLE 1

Pfmdr1 copy number of Plasmodium falciparum samples from six provinces in Thailand collected from 2002 to 2007

\begin{tabular}{|c|c|c|c|c|c|}
\hline \multirow[b]{2}{*}{ Sample location } & \multirow[b]{2}{*}{ Year } & \multirow[b]{2}{*}{$N$} & \multicolumn{3}{|c|}{ Pfmdr 1 copy number } \\
\hline & & & Single (\%) & Multiple (\%) & $\stackrel{\text { Not }}{\text { determined (\%) }}$ \\
\hline Chiang Dao & 2002 & 26 & $17(65)$ & $1(4)$ & $8(31)$ \\
\hline Chiang Dao & 2003 & 30 & $26(87)$ & 4 (13) & $0(0)^{\prime}$ \\
\hline Mae Sot & 2004 & 33 & $19(58)$ & 11 (33) & $3(9)$ \\
\hline Ranong & 2004 & 48 & 41 (85) & $0(0)$ & 7 (15) \\
\hline Phang Nga & 2005 & 44 & $29(66)$ & $2(5)$ & $13(29)$ \\
\hline Trat & 2005 & 57 & 33 (58) & $10(18)$ & $14(24)$ \\
\hline Sangkhlaburi & 2007 & 15 & 15 (100) & $0(0)$ & $0(0)$ \\
\hline
\end{tabular}

analyses of $\mathrm{K} 13$ prevalence in Thai $P$. falciparum isolates, ${ }^{11,12}$ although 3 years after $\mathrm{K} 13$ was found in neighboring Cambodia. R539T, another confirmed mutation associated with ART, was also observed in Trat. Both C580Y and R539T have been reported to be the most prevalent in Cambodia, and may have emerged in Trat via bordercrossing movements; therefore, it is possible that $\mathrm{K} 13$ mutations were present in Trat before 2005 and present in Thailand before 2004.

A recent report by Khammanee et al. 2019 also observed C580Y in samples from Ranong between 2012 and 2014 and from Phang nga in 2015, suggesting the persistence of C580Y in this area. ${ }^{8}$ The proximity of Ranong to Myanmar may also explain our observation of P574L, which is more prevalent in Myanmar. Moreover, parasites carrying C580Y allele were previously shown, via K13 microsatellites, to be distinct between Thailand-Myanmar and Thailand-Cambodia border

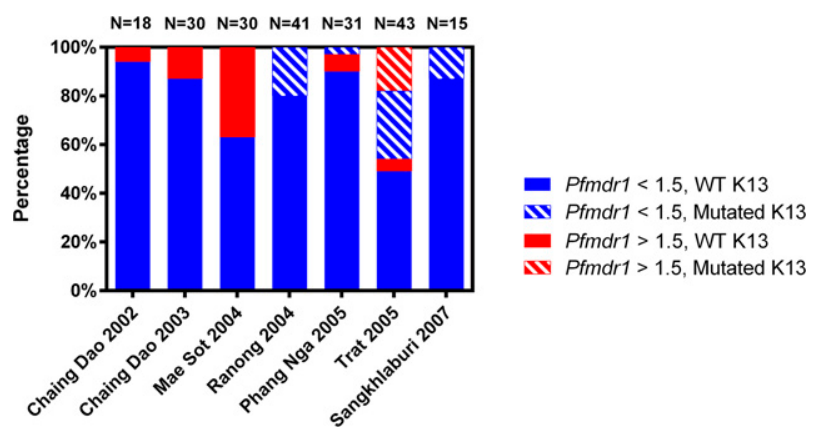

FIGURE 2. Pfmdr1 copy number and K13 mutations in Plasmodium falciparum samples in six provinces in Thailand from 2002 to 2007. This figure appears in color at www.ajtmh.org. 
areas, suggesting differences in origin and migration along these borders. ${ }^{9}$ Therefore, assessing genetic relatedness among the C580Y alleles found in Ranong, Phang Nga, and Trat would be of interest to determine the spread of these parasites across different regions of Thailand.

Multiple pfmdr 1 copies has been shown to be associated with a decrease in vitro sensitivity to mefloquine ${ }^{6}$ and it was associated with mefloquine-treatment failures in Cambodia, in which $P$. falciparum recurrent isolates had a higher pfmdr1 copies than $P$. falciparum isolates taken at the start of treatment. ${ }^{13}$ Our study showed that the highest prevalence of multiple pfmdr1 copies was found in Mae Sot, which could reflect long-term use of mefloquine monotherapy since the mid1980s. In 2016, Thailand changed malaria treatment policy from artesunate-mefloquine (AS-MQ) to dihydroartemisininpiperaquine (DHA-PIP) countrywide and the prevalence of multiple pfmdr1 copies has subsequently been shown to be declining in both eastern and western borders of Thailand. ${ }^{11}$ However, pfmdr1 copy number should be continuously monitored as the reduction of AS-MQ efficacy results from a combination of K13 mutations and multiple pfmdr1 copies. ${ }^{14}$ Cambodia changed the first-line treatment to DHA-PIP in 2012, but because of rapid loss of efficacy, switched back to AS-MQ. A similar situation was unfolding in the neighboring Thailand, and high percentages of failures to DHA-PIP were reported in Sisaket and Ubon Ratchathani provinces. ${ }^{15}$ For these regions, in 2019 the MoPH selected an alternative ACT, artesunate-pyronaridine (Pyramax), to be used, whereas DHA-PIP has remained as the treatment of the rest of the country. ${ }^{16}$ An efficacy of Pyramax is currently under evaluation in clinical trials by AFRIMS, clinicaltrials.gov identifier: NCT03726593.

Molecular evidence of ACT compromise is evident from isolates carrying both $\mathrm{K} 13$ mutations and multiple pfmdr1 copies, collected in Trat Province over 15 years ago. Unfortunately, a correlation between genotypic and phenotypic data cannot be made because of the lack of in vitro susceptibility testing of these samples. Even as national policy was shifted away from mefloquine, resistance began to mount to other partner drugs and molecular markers to drugs such as piperaquine are now in use for monitoring. If mutations become additive, such as was suggested by emergence of triple mutant parasites ( $\mathrm{K} 13$, pfmdr1, and plasmepsin 2 copy number) in Cambodia, ${ }^{17}$ a need for alternative regimens are warranted. However, ART parasites without K13 mutations have been demonstrated, suggesting that mutations in other genes may be involved. ${ }^{18}$ Mutations in pfcoronin and pfatg18 have demonstrated a reduction in ART susceptibility in non-K13-mutant parasites ${ }^{19,20}$; therefore, expanded molecular surveillance is essential for tracking resistance parasites.

In summary, this study provided retrospective information on $\mathrm{K} 13$ mutations and pfmdr1 copy number variation in $P$. falciparum isolates from different areas in Thailand dating back to 2002. Data presented confirm K13-resistance mutations arose in Thailand as early as 2004 , before the recognition of the first verified ART resistance in Thailand-Cambodia border 3 years later. The subsequent increase in proportion of isolates with $\mathrm{K} 13$ mutations and multiple pfmdr1 copies warrants continued investments in alternative therapies and continuous surveillance of resistance molecular markers with accompanying in vitro susceptibility information.
Received March 13, 2021. Accepted for publication May 14, 2021.

Published online July 16, 2021.

Acknowledgments: We would like to thank and acknowledge the study volunteers, the local malaria clinic staffs at Chiang Dao, Mae Sot, Ranong, Phang Nga, Trat, and Sangkhlaburi, and those who helped execute this study: Kanungnit Congpuong and Jeeraphat Sirichaisinthop. We also would like to thank AFRIMS malaria field team for their laboratory assistance.

Financial support: This work was supported by the United States Department of Defense, Armed Forces Health Surveillance DivisionGlobal Emerging Infectious Disease Surveillance (AFHSD-GEIS) Branch and Defense Malaria Assistance Program (DMAP). The funding source had no role in the analysis or interpretation of data, preparation of the manuscript or the decision to publish.

Disclosure: Material has been reviewed by the Walter Reed Army Institute of Research. There is no objection to its presentation and/or publication. The opinions or assertions containing herein are the private views of the author, and are not to be construed as official, or as reflecting true views of the Department of the Army or the Department of Defense.

Authors' addresses: Chaiyaporn Chaisatit, Piyaporn Sai-ngam, Sasikanya Thaloengsok, Sabaithip Sriwichai, Krisada Jongsakul, Michele Spring, Mariusz Wojnarski, Norman Waters, Paphavee Lertsethtakarn, and Brian A. Vesely, Department of Bacterial and Parasitic Diseases, Armed Forces Research Institute of Medical Sciences, Bangkok, Thailand, E-mails: chaiyapornc.ca@afrims.org, piyaporns.fsn@afrims.org, sasikanyat.ca@afrims.org, sabaithips. fsn@afrims.org, krisadaj.ctr@afrims.org, michele.spring.ctr@ afrims.org, mariusz.wojnarski.mil@afrims.org, norman.waters. mil@afrims.org, paphaveel.fsn@afrims.org, and brian.vesely.mil@ afrims.org. Mark Fukuda, Department of Retrovirology, Armed Forces Research Institute of Medical Sciences, Bangkok, Thailand, E-mail: mark.fukuda.mil@afrims.org.

\section{REFERENCES}

1. Wongsrichanalai $\mathrm{C}$, Sibley $\mathrm{CH}, 2013$. Fighting drug-resistant Plasmodium falciparum: the challenge of artemisinin resistance. Clin Microbiol Infect 19: 908-916.

2. Dondorp AM et al., 2009. Artemisinin resistance in Plasmodium falciparum malaria. N Engl J Med 361: 455-467.

3. Noedl H, Se Y, Schaecher K, Smith BL, Socheat D, Fukuda MM, 2008. Evidence of artemisinin-resistant malaria in western Cambodia. N Engl J Med 359: 2619-2620.

4. Ariey $\mathrm{F}$ et al., 2014. A molecular marker of artemisinin-resistant Plasmodium falciparum malaria. Nature 505: 50-55.

5. Ménard D et al., 2016. A worldwide map of Plasmodium falciparum K13-propeller polymorphisms. N Engl J Med 374: 24532464.

6. Price RN et al., 2004. Mefloquine resistance in Plasmodium falciparum and increased pfmdr1 gene copy number. Lancet 364: 438-447.

7. Kamau E, Alemayehu S, Feghali KC, Saunders D, Ockenhouse $\mathrm{CF}, 2013$. Multiplex qPCR for detection and absolute quantification of malaria. PLoS One 8: e71539.

8. Khammanee T, Sawangjaroen N, Buncherd H, Tun AW, Thanapongpichat S, 2019. Molecular surveillance of Pfkelch13 and Pfmdr1 mutations in Plasmodium falciparum isolates from southern Thailand. Korean J Parasitol 57: 369-377.

9. Talundzic E et al., 2015. Selection and spread of artemisininresistant alleles in Thailand prior to the global artemisinin resistance containment campaign. PLoS Pathog 11: e1004789.

10. Putaporntip C, Kuamsab N, Kosuwin R, Tantiwattanasub W, Vejakama P, Sueblinvong T, Seethamchai S, Jongwutiwes S, Hughes AL, 2016. Natural selection of K13 mutants of Plasmodium falciparum in response to artemisinin combination therapies in Thailand. Clin Microbiol Infect 22: 285.e1285.e8. 
11. Imwong $\mathrm{M}$ et al., 2020. Molecular epidemiology of resistance to antimalarial drugs in the Greater Mekong subregion: an observational study. Lancet Infect Dis 20: 1470-1480.

12. Ye R et al., 2016. Distinctive origin of artemisinin-resistant Plasmodium falciparum on the China-Myanmar border. Sci Rep 6: 20100.

13. Alker AP et al., 2007. Pfmdr1 and in vivo resistance to artesunatemefloquine in falciparum malaria on the Cambodian-Thai border. Am J Trop Med Hyg 76: 641-647.

14. Phyo AP et al., 2016. Declining efficacy of artemisinin combination therapy against $P$. falciparum malaria on the Thai-Myanmar border (2003-2013): the role of parasite genetic factors. Clin Infect Dis 63: 784-791.

15. van der Pluijm RW et al., 2019. Determinants of dihydroartemisinin-piperaquine treatment failure in Plasmodium falciparum malaria in Cambodia, Thailand, and Vietnam: a prospective clinical, pharmacological, and genetic study. Lancet Infect Dis 19: 952-961.
16. Department of Disease Control, 2019. Guideline for the Treatment of Malaria in Thailand. Available at: malaria.ddc.moph.go.th. Accessed July 31, 2020.

17. Rossi G, De Smet M, Khim N, Kindermans JM, Menard D, 2017. Emergence of Plasmodium falciparum triple mutant in Cambodia. Lancet Infect Dis 17: 1233.

18. Mukherjee A et al., 2017. Artemisinin resistance without pfkelch13 mutations in Plasmodium falciparum isolates from Cambodia. Malar J 16: 195.

19. Demas AR, Sharma AI, Wong W, Early AM, Redmond S, Bopp S, Neafsey DE, Volkman SK, Hartl DL, Wirth DF, 2018. Mutations in Plasmodium falciparum actin-binding protein coronin confer reduced artemisinin susceptibility. Proc Natl Acad Sci USA 115: 12799-12804.

20. Breglio KF et al., 2018. A single nucleotide polymorphism in the Plasmodium falciparum atg18 gene associates with artemisinin resistance and confers enhanced parasite survival under nutrient deprivation. Malar J 17: 391. 